\title{
Table of cited GATT 1947 reports
}

Belgium - Family Allowances: Belgian Family Allowances, G/32, adopted 7 November 1952, BISD 1S/5993

Canada - Alcoholic Drinks: Canada - Import, Distribution and Sale of Certain Alcoholic Drinks by Provincial Marketing Agencies, DS17/R, adopted on 18 February 1992, BISD 39S/27

Japan - Alcoholic Beverages I: Japan - Customs Duties, Taxes and Labelling Practices on Imported Wines and Alcoholic Beverages, L/6216, adopted 10 November 1987, BISD 34S/83

Sweden-AD Duties: Swedish Anti-Dumping Duties, L/328, adopted 26 February 1955, BISD 3S/81

Thailand - Cigarettes: Thailand - Restrictions on Importation of and Internal Taxes on Cigarettes, DS10/R, adopted 7 November 1990, BISD 37S/200

Tuna/Dolphin I see US-Tuna (Mexico)

Tuna Dolphin II see US-Tuna (EEC)

US - Canadian Tuna: United States - Prohibition of Imports of Tuna and Tuna Products from Canada, L/5198, adopted on 22 February 1982, BISD 29S/91

US - DISC: United States - Income Tax Legislation, L/4422, adopted 7 December 1981, BISD 23S/98

US - Malt Beverages: United States - Measures Affecting Alcoholic and Malt Beverages, DS23/R, adopted 19 June 1992, BISD 39S/206

US - Superfund: United States - Taxes on Petroleum and Certain Imported Substances, L/6175, adopted 17 June 1987, BISD 34S/136 ... 65-6, 71, 73, 78, 99, 102, 107-8, 129, 138, 228, 230

US - Taxes on Automobiles: United States - Taxes on Automobiles, DS31/R, 11 October 1994, unadopted $114,125-6$

US - Tuna (Mexico): United States - Restrictions on Imports of Tuna, DS21/R, 3 September 1991, unadopted, BISD 39S/155 ......... 86, 94, 102, 130, 164, 194

US - Tuna (EEC): United States - Restrictions on Imports of Tuna, DS29/R, 16 June 1994, unadopted 